\title{
Fast Evaluation Model of Surfactant Flooding Based on Modification Fractional Flow Theory
}

\author{
Ye Cui ${ }^{1, a}$ \\ ${ }^{1}$ No.3 Oil Production Plant of Daqing Oilfield Company Ltd, Daqing, Heilongjiang, China
}

\begin{abstract}
At present, the numerical simulation of surfactant flooding is implemented by commercial software, which requires a lot of input data, resulting in slow calculation speed and heavy workload. So it was not suitable for surfactant flooding revovery evaluation in a large number of oilfields. This paper presents a fast evaluation model of surfactant flooding based on modification fractional flow theory. Viral expansion, interfacial tension calculation formula, and the relative permeability correction model are introduced to simulate the surfactant displacement mechanism. The simulation model is applied in a low permeability reservoir, and the simulation results are compared with the eclipse software results. Their results are similar, which show that this model presents certain reliability.
\end{abstract}

\section{Introduction}

Oilfield development always pursues higher recovery. In the past few decades, Rich practical experience in onshore oilfield development has been accumulated, and the water injection development technology mainly used has also been continuously developed and perfected. In water injection development, it usually occurs that the water cut rises rapidly and the ultimate water flooding recovery rate is low. This is due to the frequent continental deposits in our country's onshore oilfields and serious heterogeneity. At the same time, with the deepening of the development of the old oilfield, it is also more difficult to adjust and, and it is difficult to further improve the water flooding recovery. In order to further improve the recovery rate after water flooding, researchers have proposed many methods, such as steam flooding, surfactant flooding, microbial flooding and foam flooding. Then how to evaluate the potential of different methods to improve oil recovery has become a problem that needs to be solved.

\section{Research Status of Rapid Evaluation Software for Enhanced Recovery Potential}

Numerical simulation technology can reasonably calculate the expected effects of different enhanced oil recovery development plans, and on this basis, the potential of different enhanced oil recovery methods can be evaluated, so as to effectively guide the formulation of development plans. The advantages of numerical simulation technology are obvious: the simulation cost is relatively low, the simulation can be repeated, and the overall oil field can be evaluated. At present, there are

a Corresponding author: zhangbin1018@petrochina.com.cn many mature commercial numerical simulation software, which is powerful and can simulate the development of most oil reservoirs. However, these softwares also have some limitations: simulation calculation needs to involve some parameters that are not easy to obtain in actual oil field development, the construction of geological models is time-consuming and laborious, and computational simulation requires a lot of time. Therefore, it is necessary to design a rapid evaluation model for enhanced oil recovery, using a small number of parameters that are easy to obtain in oil fields, and abandoning complex geological models.

Currently, the main enhanced oil recovery (EOR) evaluation models include compositional, black oil, streamline, and fractional flow theory models. The compositional model can achieve reliable computation results and effectively simulate seepage characteristics of injected solvent and crude oil. However, it requires detailed physical data of rock and fluid, complex computation, and heavy workload. Compared with the compositional model, the black oil model has higher computing speed and requires less data, but it still needs considerable physical data of rock and fluid for support. Black oil model is composed of a set of nonlinear parabolic partial differential equations, auxiliary equations, boundary conditions and initial conditions. Since the initial conditions are used to describe the initial state of reservoir development, its accuracy plays a key role in describing the dynamics of reservoir development. The streamline model is a simulation method derived from the black oil model or compositional model, which presents improved computing speed by transforming 3D flow to 1D flow. However, it still has strict requirements on data. The fractional flow theory model features a fast computing speed and low data demand by transforming $3 \mathrm{D}$ flow to $1 \mathrm{D}$ flow, but it simplifies some parts of the 
seepage characteristics [1-3]. At present, a large number of oilfields are conducting research on EOR mechanism and feasibility. At the EOR evaluation stage of an oilfield, each oilfield comprises hundreds or thousands of different oil reservoirs, resulting in heavy work in EOR potential evaluation, which indicates the importance of a fast and reliable EOR evaluation model. This paper established corresponding EOR evaluation models by adopting porous media seepage theory, reservoir numerical simulation technology, and numerical computation method based on fractional flow theory. Necessary modifications were made to the fractional flow theory model according to surfactant flooding mechanisms.

\section{Basic principles of the fractional flow theory evaluation model}

The basic assumptions of the fractional flow theory model are as follows: three phases are considered, namely, oil, gas, and water; flow of each phase follows Darcy's Law; displacement is an isothermal process; viscous fingering is described with Koval coefficient; when injecting by alternate displacement, the injectants are injected simultaneously at a fixed proportion; no free gas exists; no large cracks in oil reservoir or leakage of injected gas occurs; adsorption follows mass adsorption law, and the process is irreversible; and diffusion and dispersion are considered [4-6].

The equation of mass conservation of fractional flow theory is

$$
\frac{\partial C_{i}}{\partial t_{D}}+\frac{\partial F_{i}}{\partial X_{D}}=0
$$

where

$\mathrm{i}=1$-water component;

$\mathrm{i}=2$ - crude oil component;

$\mathrm{i}=3$ - injected gas component;

$$
\begin{aligned}
& X_{D}=\mathrm{X} / \mathrm{L} \text { _dimensionless distance; } \\
& t_{D}=\int_{0}^{t} q d t / d V_{p} \text { _dimensionless }
\end{aligned}
$$

$\mathrm{Fi}$ - total fractional flow of component $\mathrm{i}$; $\mathrm{Ci}$ - total concentration of component $i$, which can be expressed as

$$
\begin{array}{cc}
C_{i}=C_{i 1} S_{j}+C_{i 2} S_{2}+C_{i 3} S_{3} & \mathrm{i}=1,2,3 \\
F_{i}=C_{i 1} f_{j}+C_{i 2} f_{2}+C_{i 3} f_{3} & \mathrm{i}=1,2,3
\end{array}
$$

where

$C_{i j}$ _concentration of component $\mathrm{i}$ in phase $\mathrm{j} ; \mathrm{j}=1-$ water phase; $\mathrm{j}=2$-oil phase; $\mathrm{j}=3$-injected gas phase;

$S_{j}$

${ }_{j}$ - saturation of phase $\mathrm{j}$;

$f_{j}$ _fractional flow of phase $\mathrm{j}$.

If injected gas and crude oil achieve miscible displacement, Equations (2) and (3) are modified as a two-phase conservation equation:

$$
\begin{gathered}
C_{i}=C_{i j} S_{j}+C_{i 2} S_{2} \\
F_{i}=C_{i j} f_{j}+C_{i 2} f_{2}
\end{gathered}
$$

The partial differential equation can be further expressed as

$$
\frac{\partial C_{i}}{\partial t_{D}}+\left(\frac{\partial F_{i}}{\partial C_{i}}\right)_{X_{D}} \cdot \frac{\partial C_{i}}{\partial X_{D}}=0
$$

A concentration ratio (fixed) is defined and expressed as $\left(\frac{\partial F_{i}}{\partial C_{i}}\right)$ ; under compatible condition and the same concentration, all the components have equal concentration ratio.

$$
V_{c i}=d F_{i} / d C_{i} \equiv \lambda, \quad \mathrm{i}=1,2,3
$$

Thus, the two rates can be expressed as follows:

$$
\begin{aligned}
& \lambda \pm=0.5\left\{\mathrm{~F}_{22}+\mathrm{F}_{33} \pm\left[\left(F_{22}-F_{33}\right)^{2}+4 F_{32} \cdot F_{23}\right]^{1 / 2}\right\} \\
& \mathrm{F}_{23}=\left(\frac{\partial F_{2}}{\partial C_{3}}\right)_{C_{2}} \text {, etc. }
\end{aligned}
$$

Rate $\lambda \pm$ is defined as two similar component lines (direction): fast and slow lines. Fast line must pass the initial conditions (in the oil reservoir), whereas slow line has to pass the injection conditions [7-8]. The model first goes through two-phase flash calculation and fractional flow computation along the component lines. After the fast and slow lines are determined, their point of intersection where the component lines transfer from the fast line to the slow line is determined (Figure 1). According to the fast and slow lines of concentration, the concentration and fractional flow of fluid within the affected range of gas flooding can be obtained, as well as the oil well production and oil reservoir recovery.

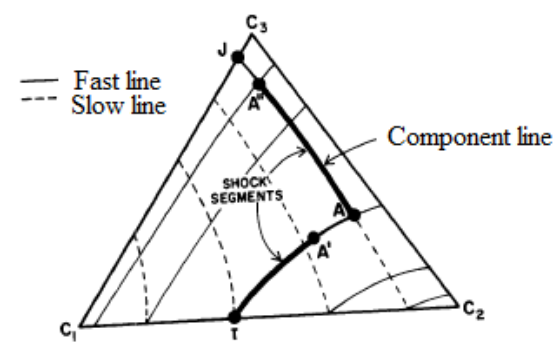

Figure 1. Phenogram of fast and slow lines

\section{Modification of surfactant flooding seepage models}

The surfactant flooding mechanism of surfactant flooding mainly includes reducing the oil-water interfacial tension and improving displacing efficiency; emulsification mechanism: under the flowing and shearing of oil and water phases, crude oil on the surface of rock can quickly disperse and strip, forming an oil-inwater $(\mathrm{O} / \mathrm{W})$ emulsion and improving the mobility ratio of oil and water phases; changing the wettability of the rock surface, and suitable surfactants can increase wetting contact angles between crude oil and rock, making the rock surface shift from oil wet to water wet 
Focusing on surfactant adsorption, crude oil emulsification, interfacial tension reduction, and other issues, this study modified fractional flow theory.

(1) Adsorption

The equation of calculating the adsorption ratio (U.S. Department of Energy) is applied as follows:

$$
D S=\left(\frac{1-\phi}{\phi}\right) \cdot \frac{\rho_{\text {rock }}}{\rho_{\text {surf }}} \cdot \frac{A_{s}}{C_{s}} / 1000
$$

where

DS is adsorption coefficient;

$\phi$ is porosity;

$\rho_{\text {rock }}$ is rock density $(\mathrm{g} / \mathrm{ml})$;

$\rho_{\text {surf }}$ is foam density $(\mathrm{g} / \mathrm{m})$;

AS is proportion of clay mineral (no unit); $C_{s}$ is foam concentration (no unit).

(2) Modify the viscosity of water (surfactant is dissolved in water, thereby changing the viscosity of water)

Applying virial expansion,

$$
\mu_{\text {surf }}=\mu_{w}+K_{\text {pol }} C_{\text {surf }}
$$

where

$K_{p o l}$ is constant (no unit);

$C_{s \text { is surfactant concentration; }}$

$\mu_{w}$ is the viscosity of water.

(3) Interfacial tension calculation model

Apply Schechter's computation model [7] as follows:

$$
\log \left(\sigma_{o s}\right)=\log \left(\sigma_{\min o s}\right)+\left[\log \left(\frac{\sigma_{\max o s}}{\sigma_{\min o s}}\right)\right]\left(\frac{C_{\sigma_{\max o s}}-C_{\sigma o s}}{C_{\sigma_{\max } o s}-C_{\sigma_{\min } o s}}\right)^{e s}
$$

$\sigma_{o s}, \sigma_{\text {minos }}$, and $\sigma_{\text {maxos }}$ are instantaneous interfacial tension, minimum interfacial tension, and maximum interfacial tension between the oil surfactants, respectively; $C_{\sigma}, C_{\sigma_{\min }}$, and $C_{\sigma_{\max }}$ are surfactant's instantaneous concentration, the concentration at minimum interfacial tension, and the maximum interfacial tension, respectively; and $e s$ is exponent parameter [9].

(4) Modification model of relative permeability

Based on the determination of interfacial tension, this paper introduced the relative permeability's modified coefficient $\mathrm{F}$, through which relative permeability was modified.

$$
\begin{gathered}
F=\frac{\sigma_{o s}}{\sigma_{r e f}} \\
S_{\text {oros }}=S_{o r} \cdot F \\
k_{r}=F \cdot k_{r}^{o w}+(1-F) k_{r}^{m}
\end{gathered}
$$

where

$F$ : relative permeability's modified coefficient (no unit); $\sigma_{o s}$ :interfacial tension of oil and surfactant;

$\sigma_{r e f}$ :referenced interfacial tension;

$S_{\text {oros }}$ : residual oil saturation after surfactant injection;

$k_{r}$ : oil-phase relative permeability after surfactant injection;

$k_{r}^{o w}$ : the original oil-phase relative permeability without surfactant injection; and

$k_{r}^{m}$ : oil-phase relative permeability when the interfacial tension is 0 .

\section{Evaluation and Discussion}

Using a typical low permeability reservoir as an example, the modified fractional flow theory model and software Eclipse were used to predict the EOR potential of the oil reservoir. The comparison of predicted results is shown in Figures 2. The calculated results of the modified model are close to that of commercial software Eclipse with a difference within $10 \%$ on average, which implies a good effect. However, the computation speed of the fractional flow theory calculation model is much faster than that of Eclipse [10-11].

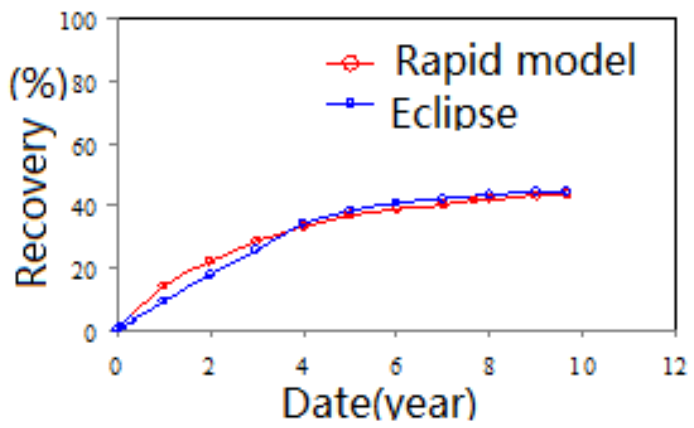

Figure 2. EOR effect contrast of surfactant flooding

The main reason for the difference between the two is that Eclipse adopts the finite difference method and considers factors, such as capillary effect. Besides, the numerical simulation model of Eclipse shows heterogeneity of oil reservoirs, but the fractional flow theory model merely adopts numerical treatment for heterogeneity. The other mechanisms are similar. A comparison of results between the modified fractional flow theory model and Eclipse shows that the computational accuracy of the modified fractional flow theory model can satisfy preliminary evaluation on large-scale EOR for all oil reservoirs. Moreover, the model can provide technical support for EOR technology implementation and economic evaluation of an entire oil field [12-14].

\section{Conclusion}

This paper presents a fast evaluation model of surfactant flooding based on modification fractional flow theory. Viral expansion, interfacial tension calculation formula, 
and the relative permeability correction model are introduced to simulate the surfactant displacement mechanism. The comparison with results of Eclipse shows that the modified EOR prediction model has a high calculation speed and reliable results, so it can be applied to evaluate an oil field's EOR potential through gas flooding.

\section{References}

1. Kim J S, Mobil E. PUS Inc, Compositional Simulation of the Coyanosa Wolfcamp Field Gas Cycling Operation[J]. SPE20130, 1990.

2. Libing Gu, Zhiping Li. The prograss of CO2 EOR [J]. China Mining. 2007(10): 66-69.

3. C. Liao, et al., Correlations of minimum miscibility pressure for pure and impure $\mathrm{CO} 2$ in low permeability oil servoir, Journal of the Energy Institute (2014), http://dx.doi.org/10.1016/j.joei.2014.03.012

4. Vargo J, Turner J, Bob V, et al. Alkaline-surfactantpolymer flooding of the Cambridge Minnelusa Field[J]. SPE Reservoir Evaluation \& Engineering, 2000, 3(06): 552-558.

5. Elraies K A, Tan I. Design and application of a new acid-alkali-surfactant flooding formulation for Malaysian reservoirs. SPE 133005, presented at the SPE Asia Pacific oil and gas conference and exhibition[J]. 2010.

6. Dirk J. Groenendijk, Ron Bouwmeester, Johannes N. M. van Wunnik. Spectrophotometric Determination of $\mathrm{Ca} 2+$ and $\mathrm{Ca}-C o m p l e x$ Formation Constants: Application to Chemical Enhanced Oil Recovery. ACS Omega 2021, 6 (7), 5027-5032.

7. Jan Kubelka, Shixun Bai, Mohammad Piri. Effects of Surfactant Charge and Molecular Structure on Wettability Alteration of Calcite: Insights from Molecular Dynamics Simulations. The Journal of Physical Chemistry B 2021, 125 (4) , 1293-1305.

8. Zongyang Li, Yefei Wang, Hong He, Fuqing Yuan, Haocheng Liu, Yuqiu Chen. Insights into the Effects of Salinity on the Transport Behavior of PolymerEnhanced Branched-Preformed Particle Gel Suspension in Porous Media. Energy \& Fuels 2021, 35 (2) , 1104-1112.

9. Liu, C. (1990) Well test analysis method of non Newtonian fluid for vertically fractured wells with limited conductivity in dual porosity media. Acta Petrolei Sinica, 11 (4) : 61-67.

10. Li,J., Wu, X., Zhao, L. A well logging method for evaluation effect of drilling mud on formation contamination. Xinjiang Petrol Geol, 2010;31(3):318-319.

11. LI Xingguo. Exploration and Development of Oilfield in Middle and High Water Cut Period. Petroleum Exploration and Development. Geological Publishing House. 1991.
12. Grigg R, siagian U.Understanding and Exploiting Four-Phase Flow in Low-Temperatue $\mathrm{CO}_{2}$ Floodings.1998.

13. Claridge E L.Discussion of zhe Use of Capillary Tube Networks in Reservoir Performance Studies[J]. Society of Petroleum Engineers Journal. 1972, 12(4): 352-361.

14. Welge H J, Williams P S. Preventing water encroachment in oil wells[C]. US, 1952. 